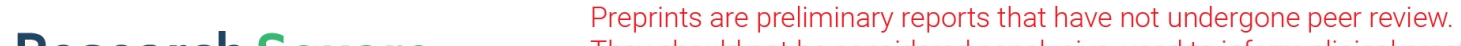 They should not be considered conclusive, used to inform clinical practice, or referenced by the media as validated information. \\ On Channel Estimation and Power Control for Multi- cell Multi-path Massive MIMO TDD System
}

Jamal AMADID ( $\square$ jamal.amadid@edu.uca.ac.ma )

Universite Cadi Ayyad Faculte des Sciences Semlalia https://orcid.org/0000-0002-6158-069X

Abdelfettah Belhabib

Universite Cadi Ayyad Faculte des Sciences Semlalia

Mohamed Boulouird

Cadi Ayyad University Normal Graduate School of Marrakech: Universite Cadi Ayyad Ecole Normale Superieure de Marrakech

Moha M'Rabet Hassan

Universite Cadi Ayyad Faculte des Sciences Semlalia

Abdelouhab Zeroual

Universite Cadi Ayyad Faculte des Sciences Semlalia

\section{Research Article}

Keywords: Massive MIMO, Channel Estimation, Pilot Contamination, Multi-path Channels, Maximum likelihood, Power Control

Posted Date: January 7th, 2022

DOI: https://doi.org/10.21203/rs.3.rs-454402/v1

License: (c) (i) This work is licensed under a Creative Commons Attribution 4.0 International License.

Read Full License 
Noname manuscript No.

(will be inserted by the editor)

\title{
On Channel Estimation and Power Control for Multi-cell Multi-path Massive MIMO TDD System
}

\author{
Jamal Amadid ${ }^{1}$. Abdelfettah Belhabib ${ }^{1}$. \\ Mohamed Boulouird $^{2}$. Moha M'Rabet \\ Hassani $^{1}$. Abdelouhab Zeroual ${ }^{1}$
}

Received: date / Accepted: date

\begin{abstract}
Some more practical channels that model the networks in a real environment is the multi-path communication channels. In order to investigate these communications channels. This work addressed Channel Estimation (CE) in the Uplink (UL) phase for a multi-cell multi-user massive multipleinput multiple-output (M-MIMO) system that studies multi-path communication between each user and its serving Base Station (BS). We suppose that the network operates under Time-Division Duplex (TDD) protocol. We studied and analyzed the multi-path channels and their benefit over CE since it presents a more realistic channel that displays a real propagation circumstance. on the flip side, we evaluated the CE quality using ideal MinimumMean Square Error (MMSE). This latter relies on an impractical property that can be explicated since the MMSE estimator considers foreknowledge on Large-Scale Fading (LSF) coefficients of interfering users. Thus, the suggested estimator is introduced to overcome this issue, where the suggested estimator tackled this problem and presented result asymptotic approaches to the performance of the MMSE estimator. Besides, we considered a more real communication in which the multi-path channels are either realized using Non-Line-of-Sight (NLoS) only or using both Line-of-Sight (LoS) and NLoS path depending on the distance at which the user is located from his serving BS. Otherwise, in numerous scenarios, users at the cell edge are strongly af-
\end{abstract}

Jamal Amadid

E-mail: jamal.amadid@edu.uca.ac.ma

Abdelfettah Belhabib

Mohamed Boulouird

Moha M'Rabet Hassani

Abdelouhab Zeroual 
fected by Pilot Contamination (PC). Hence, we introduced a Power Control (PoC) policy so that the users at the cell edge are less affected by the PC problem. In the simulation results segment, the analytic and simulated results are introduced to assert our theoretical study.

Keywords Massive MIMO - Channel Estimation · Pilot Contamination · Multi-path Channels · Maximum likelihood · Power Control

\section{Declarations}

Funding : Not applicable

Conflicts of interest/Competing interests : Not applicable

Availability of data and material : Not applicable

Code availability : Not applicable

Authors' contributions : Not applicable

\section{Acronyms}

BS : Base Station

CE : Channel Estimation

CSI : Channel State Information

DL : Downlink

EE : Energy Efficiency

MMSE : Minimum Mean Square Error

LS : Least-Squares

LoS : Line of Sight

LSF : Large-Scale Fading

M-MIMO : Massive Multiple Input Multiple Output

ML : Maximum likelihood

MSE : Mean Square Error

NLoS : Non-Line of Sight

NoA : Number of Antennas

NoAE : Number of Averaged Elements

OFDM : Orthogonal Frequency-Division Multiplexing

PC : Pilot Contamination

PE : Polynomial Expansion

PoC : Power Control

PS : Pilot Sequence

SE : Spectral Efficiency

SNR : Signal-to-Noise Ratio

SSF : Small-Scale Fading

TDD : Time Division Duplex 
UL : Uplink

\section{Introduction}

Massive Multiple Input Multiple Output (M-MIMO) considers as a promising technology that brings many enhancements to the wireless communication network, which relies on the employ of hundreds of antennas in each Base Station (BS) that allows benefiting from beamforming scheme that increases the communication gain and focuses the signal in the desired region 1, 2. Besides, M-MIMO technology give rise to a considerable enhancement over Spectral Efficiency (SE) and Energy Efficiency (EE) by using linear processing schemes. The enhancement in SE is made through the service of many terminals at the same time-frequency resources using a spatial multiplexing technique, while the boost in EE is obtained through the use of a massive Number of Antennas (NoA) at the BS [3, 4, 5]. To achieve great improvements in M-MIMO systems, a precise channel statistics are necessary to be know at both end (i.e., the transceiver) for detecting and pre-coding purposes. In addition, the reuse of Pilot Sequences (PSs) (i.e., frequencies ) in cellular networks degrading the accuracy of Channel Estimation (CE). In other words, the reuse of pilot/frequencies in cellular networks give rise to a bottleneck phenomenon for the M-MIMO called in the literature as the Pilot Contamination (PC) 6, 7]. On the other hand, in literature, Time-Division Duplex (TDD) mode is the most appropriate protocol for the execution of M-MIMO systems, which counteracts pilot overhead by relying on channel reciprocity between the Uplink (UL) and Downlink (DL) ( i.e., the pilot signals are dispatched just in the UL phase ), as stated in the literature [7,8, 9]. In many works, the Minimum Mean Square Error (MMSE) [10,11] and Least Square (LS) [12] estimators are used to estimate the channel, which requires an accurate Channel State Information (CSI) at the BS. Besides, the MMSE estimator is relying on the property of having complete knowledge of inter-cell and intra-cell LargeScale Fading (LSF) coefficients, which is an unrealistic property as it cannot be practically applicable. The CE process considering the flat fading channel has been addressed in numerous studies in the relevant literature [6, 13, 14, 15], which only concern the Orthogonal Frequency-Division Multiplexing (OFDM) schema and do not represent a practical communication that reflects the actual communication environment. [16].

The subsection below is introduced to provide some related works, in order to position our work according to previous ones.

\subsection{Related works}

In this segment, we look at previous studies that have been tackled CE and $\mathrm{PC}$ alleviation. 
In [3], the authors deal with the LS estimator to investigate and assess the effect of PC over such systems. They deduce that using several ensembles of orthogonal training sequences in different cells leads to a relatively small difference regarding the signal-to-interference ratio.

An MMSE-based precoding method is proposed in [6], in which the problem of PC is addressed for a multicell M-MIMO system. By consideration of an ensemble of PSs allocated to users in order to get the solution of an optimization problem, the authors have minimized the error perceived by users in the hosted cell and the interference perceived by users in other cells.

In [12, the authors treated the spatially correlated channels for M-MIMO systems. In particular, they built a CE covariance-aided method that incorporates both desired and interfering channel covariance. The Bayesian approach is employed to determine two channels estimators, where the first is dealing with all channels in the network to the target cell, while the second is deals just with the channels from users in the target cell. They concluded that, in the ideal situation, the covariance of desired and interference channels are distinguished ( i.e., covering separate subspaces). In this case, the PC problem tends to disappear as the NoA at the BS increase.

A low complexity MMSE estimator based on Polynomial Expansion (PE) is proposed in [17, which offers a reduced complexity compared to the traditional MMSE estimator. The proposed MMSE estimator provides a square complexity, while the traditional MMSE estimator has a cubic complexity since it relies on a matrix inversion that increases the computational complexity. The suggested PE based MMSE estimator provides an almost-optimal Mean Square Error (MSE) even if a low degree of PE is chosen.

The authors in [18 addressed the spatially correlated channel, which uses the exponential correlation model to describe the correlation across channels. An approximate MMSE estimator is suggested based on a sample covariance matrix, wherein the efficiency of the suggested estimator is attached to the number of samples used to estimate the actual covariance matrix. Thus, The suggested estimator precision is a direct result of the number of samples used to estimate the true covariance matrix since using the suggested estimator with a massive number of samples covariance matrix provides the same result as the Bayesian-MMSE estimator.

In [19], the authors address the spatially correlated channels employing Rician fading, where the channels are a composite of deterministic (i.e., Line-of-Sight (LoS) path) and stochastic (i.e., Line-of-Sight (NLoS) path) components. The authors focused on spectral efficiency using three estimators (MMSE, ElementWise MMSE, and LS estimators). Concluding that spatial correlation improves spectral efficiency, and a significant improvement is observed over spectral efficiency when the LoS path is available.

The literature review indicated that, in general, the works that have been discussed on $\mathrm{CE}$ and $\mathrm{PC}$ assume perfect knowledge of inter/intra-cell LSF coefficients, which is not the case in practical implementations. However, researchers in the literature propose strategies to overcome this problem (i.e., 
PC). However, these strategies require further computation. Thereby, a higher complexity is provided.

\subsection{Work-organization}

This work is organized as follows:

Throughout section 2, the system model is presented, wherein we define the channel coefficients, the LSF expressions, the pilot-type used for the UL phase, and the strategy of PoC policy. In section 3, we define the CE phase and the estimators employing during this work. Simulation results are presented in section 4 to prove our theoretical study. The conclusion is provided in section 5, in which we summarize the results of the work.

\subsection{Contributions}

This work aims to study CE for M-MIMO systems running under TDD mode using Power Control (PoC) for the multi-path channel model. Furthermore, the multi-path channels can be realized either using NLoS only or using LoS and NLoS path depending on the distance from the user to its serving BS.

The main contributions are three-fold listed as follows :

1. Suggest a strategy based on Maximum-likelihood to overcome the unrealistic property afforded through the MMSE in a scenario where the communication user-BS can be made using either NLoS only or using LoS and NLoS path depending on the distance from the user to its serving BS.

2. Investigate and analyze a multi-path channel model in which the LSF has two expressions depending on the distance from the user to its serving BS (i.e., depending on whether the LOS path exists or not).

3. The employ of PoC so that the users within the cell-edge can be affected by lower interference from users in contiguous cells ( since we reflected the worst situation in which the pilot sequences employed in each cell are duplicated in the entire remaining cells ). Where, in many scenarios, users in the cell edge are strongly affected by PC, which degrades the CE quality.

\section{System model}

In this segment, we define the system model that will be utilized herein. In which L hexagonal-contiguous cells are employed. Where each cell contains $\mathrm{K}$ users with a single antenna each and a centered BS (which obey-to the constraints stated by the M-MIMO technology (i.e., $M>>K)$ ). Furthermore, each BS is armed with $\mathrm{M}$ antennas operating under the TDD protocol that has a significant advantage over the M-MIMO system as mentioned in [3, 5, 9, The service of users in any cell is provided according to the principle of timefrequency block. on the flip side, another constraint is the coherence block 
limitation which also limits the duration of the TDD frame that depends on the propagation environment (velocity of users, number of wavelengths, ....) [5, $9,20,21$.

Obeying to the TDD framework and assumed synchronized-users transmission. First, the users of all cells dispatch their training/pilot sequences in the UL process. After, the BS exploits these PSs to perform the CE process. Then, the users of all cells dispatch the payload data. Later, based on the estimated channel vectors, each BS is activated for detection and decoding the UL user data and produce precoding vectors to carry out the downlink data.

We suppose that this work is considered wireless channels that encompass both channel coefficients ( i.e., small-scale/fast-scale fading and LSF coefficients). In addition, the wireless channel employed is assumed to be static in each coherence block and doesn't rely on the BS antennas, M and number of users per cell, K. We start our process of derivation through the following explanation of $h_{j k}^{i m(p)}$ ( is the $p_{t h}$ component of $\mathbf{h}_{j k}^{i m}=\left[h_{j k}^{i m(0)}, h_{j k}^{i m(1)}, \ldots, h_{j k}^{i m(P-1)}\right]$ ) which symbolizes the gain of the $p_{t h}$ path joining the $k_{t h}$ user in the $j_{t h}$ cell to the $m_{t h} \mathrm{BS}$ antennas in the $i_{t h}$ cell. This gain is a function of both the fast-scale fading and LSF coefficients, as indicated in the equation below

$$
h_{j k}^{i m(p)}=\sqrt{\beta_{j k}^{i}}\left(g_{j k}^{i m}\right)^{(p)},
$$

Here, $\left(g_{j k}^{i m}\right)^{(p)}$ describe the rapid fluctuation in the amplitude-phase of the signal and $\beta_{j k}^{i}$ describe the path-loss or signal attenuation over a wide geographical area. Besides, assuming that the propagation environment provides at most $\mathrm{P}$ paths. The matrix that englobes/incorporates all the channel vectors $\mathbf{H}_{i j}$ is formulated as

$$
\begin{aligned}
& \mathbf{H}_{i j}=\left[\begin{array}{cccc}
\mathbf{h}_{j 1}^{i 1} & \mathbf{h}_{j 2}^{i 1} & \ldots & \mathbf{h}_{j K}^{i 1} \\
\mathbf{h}_{j 1}^{i 2} & \mathbf{h}_{j 2}^{i 12} & \ldots & \mathbf{h}_{j K}^{i 2} \\
\mathbf{h}_{j 1}^{i 3} & \mathbf{h}_{j 2}^{i 3} & \ldots & \mathbf{h}_{j K}^{i 3} \\
\vdots & \vdots & \ddots & \vdots \\
\mathbf{h}_{j 1}^{i M} & \mathbf{h}_{j 2}^{i M} & \ldots & \mathbf{h}_{j K}^{i M}
\end{array}\right] \\
& \mathbf{H}_{i j}=\left[\mathbf{H}_{j 1}^{i}, \mathbf{H}_{j 2}^{i}, \ldots, \mathbf{H}_{j K}^{i}\right]
\end{aligned}
$$

This matrix encompasses the entire channels from the $\mathrm{K}$ users in $j_{\text {th }}$ cell to all M BS antennas in $i_{t h}$ cell, where $\{i, j\}=1, \ldots, L$.

Multipath channels are a more realistic propagation environment that occurs in any communication network and are mostly obtained in urban micro ( in which large buildings are more likely to exist, and the distance between either pair of BSs is less than $1 \mathrm{~km}$, as stated in 22]). These multi-path channels are the principal effect of Small-Scale Fading (SSF) 23], where multipath 
channels depend on the environment ( propagation delays, the distance between Transmitter-Receiver,........). Thereby, this work aims to investigate and analyze a multipath channel model in which the LSF has two expressions depending on the distance between the user and BS (i.e., Line-of-Sight (LoS) or Non-Line-of-Sight (NLoS)). The LoS and NLoS path's existence depends on the distance at which the user is located, as stated in the 3 GPP model given in 22. Meaning that there is a maximal distance at which the LoS path possibly would not exist. In other words, there is an environment-dependent distance for which LoS paths are not reasonable (where all of the paths are main NLoS ) and a distance for which NLoS and LOS paths are available. The LoS probability depends on the distance between the user and its serving BS according to the following equation

$$
\operatorname{Pr}(L o S)= \begin{cases}\frac{300-d_{j k}^{i}}{300}, & 0<d_{j k}^{i}<300 \\ 0, & d_{j k}^{i}>300\end{cases}
$$

The existence of the LoS or NLoS path influences the LSF expression. Thereby, if there is a LoS path between transmitter and receiver (where all of the paths are main NLoS ), the appropriate LSF coefficient is expressed (in $\mathrm{dB}$ ) as follows

$$
\beta_{j k}^{i}=-30.18-10 \alpha^{\operatorname{LoS}} \log _{10}\left(d_{j k}^{i}\right)+\Upsilon_{j k}^{i},
$$

Here, $\Upsilon_{j k}^{i} \sim \mathcal{N}\left(0,\left(\sigma_{s f}^{L o S}\right)^{2}\right)$ signifies the shadowing or shadow fading with $\sigma_{s f}^{L o S}=$ 4 is determining how the extent of the random fluctuations are. If there isn't a LoS path between transmitter and receiver, the appropriate LSF coefficient is formulated (in $\mathrm{dB}$ ) as

$$
\beta_{j k}^{i}=-34.53-10 \alpha^{N L o S} \log _{10}\left(d_{j k}^{i}\right)+\Psi_{j k}^{i},
$$

Here, $\Psi_{j k}^{i} \sim \mathcal{N}\left(0,\left(\sigma_{s f}^{N L o S}\right)^{2}\right)$ signifies the shadowing with $\sigma_{s f}^{N L o S}=10$. After describing the nature of the LSF used in our work, the UL training phase is the next part that will be addressed.

\subsection{Uplink Training phase}

We deal with pilot-based estimation in this work. Thereby, in this stage, users in each cell dispatch pilot/training sequences in order to communicate with the desired BS. Let us assume that the reuse of frequency across the cells is one ( due to the limited coherence block), which is the worse scenario in which the system suffers from strong PC ( which represents a bottleneck for M-MIMO systems even if the NoA at the BS reach infinity). Besides, we assume the presence of synchronizing-time among cells. All these hypotheses reflect the worst possible situation regarding the M-MIMO [3]. The PS dispatched by the $k_{t h}$ user is formulated as follows

$$
\phi_{k}=\left[\psi_{k}(0), \psi_{k}(1), \psi_{k}(2), \ldots, \psi_{k}(N-1)\right]
$$


Here, $\mathrm{N}$ refers to the length of the PSs. As we investigated the multi-path channel, we consider that each communication between a user and a BS antenna is realized using the $\mathrm{P}$ paths. In addition, the convolution between the PSs dispatched by the $k_{t h}$ user and the $\mathrm{M}$ channels linking this user to a given BS can be matrix-expressed as follows

$$
\Phi_{k}=\left[\begin{array}{cccc}
\psi_{k}(0) & \psi_{k}(N-1) & \ldots & \psi_{k}(N-P+1) \\
\psi_{k}(1) & \psi_{k}(0) & \ldots & \psi_{k}(N-P+2) \\
\psi_{k}(2) & \psi_{k}(1) & \ldots & \psi_{k}(N-P+3) \\
\vdots & \vdots & \ddots & \vdots \\
\psi_{k}(N-1) & \psi_{k}(N-2) & \ldots & \psi_{k}(N-P+N)
\end{array}\right]
$$

This matrix $\left(\Phi_{k} \in \mathbb{C}^{N \times P}\right)$ is built based on a PS circular-shifted of the $k_{t h}$ user. As has been stated in the literature [Section 7 in [24], [25, 26, 27], each component of this matrix can be determined as follows

$$
\psi_{k}(n)=e^{-i \pi f \frac{n(n+1)}{N}}, \quad n=0, \ldots, N-1
$$

Here, $f$ refers to the sequence index, which obeys the constraint, being a prime number relative to the PS length $N$ and $n$ take values from 0 to $\mathrm{N}-1$ (i.e., length of PSs ). Additionally, The matrix given in (7) adapted to the following orthogonal property $\Phi_{k}^{H} \Phi_{k}=N \mathbf{I}_{P}$. It is interesting to note that, in the cyclically shifted version, these PSs offer an orthogonal property to each other [25. Furthermore, a global matrix ( $\boldsymbol{\Phi} \in \mathbf{C}^{N \times K P}$ ) can be defined, which include the PSs of $\mathrm{K}$ users and can be represented by

$$
\boldsymbol{\Phi}=\left[\Phi_{1}, \Phi_{2}, \ldots, \Phi_{K}\right]
$$

The intra-cell interference is not covered in this work. Thereby, each user of the $\mathrm{K}$ users in each cell must employ an orthogonal pilot compared to the remaining users (i.e., the length of PSs must be superior to the number of users in each cell times the number of paths, $N>K P$ ). As mentioned beforehand, the PS length must be a prime number and very close to $K \times P$.

In the situation where $N>K P L$ is proved. Thus, the system can never suffer from the PC problem. As each user uses a unique PS (i.e., orthogonal PS to all other PSs used in the network) that does not reflect the practical implementation due to the constraint given by the limiting coherence block. The BS in the $i_{t h}$ cell receives the signal $Y \in \mathbb{C}^{M \times N}$, which can be written as

$$
\mathbf{Y}_{i}=\sum_{j=1}^{L} \sum_{k=1}^{K} \sqrt{q}_{j k} \mathbf{H}_{i j k} \Phi_{k}^{T}+W_{i}
$$

Here, $q_{j k}$ is symbolized the transmit power dedicated to the $k_{t h}$ user in the $j_{\text {th }}$ cell, and $W_{i} \in \mathbb{C}^{M \times N}$ symbolizes the noise matrix related to the $i_{t h}$ BS with the property that each element of $W_{i}$ is following the normal distribution (i.e., $\mathcal{C N} \sim(0,1)$ ).

Before the estimation stage, we can write the equation 10 without depending 
on the PS. Hence, the equation (10) can be rewritten in a new form (as a function of channels and noise only ) as follows

$$
\mathbf{y}_{i k}=\mathbf{Y}_{i} \Phi_{k}^{*}=N \sum_{j=1}^{L} \sqrt{q_{j k}} \mathbf{H}_{i j k}+w_{i k}
$$

Where $\mathbf{y}_{i k}$ is a $M \times 1$ vector, and $w_{i k}=W_{i} \Phi_{k}^{*}\left(\in \mathbb{C}^{M \times 1}\right)$ is represented as $\mathcal{N}\left(0_{M \times 1}, \mathbf{I}_{M}\right)$.

\subsection{Power Control Strategy}

We have introduced the heuristic PoC [9] in order to users in the cell-edge can be affected by lower interference from users in contiguous cells. The transmit power devoted to the $k_{t h}$ user in the $i_{t h}$ cell can be expressed as follows

$$
q_{i k}=\left\{\begin{array}{l}
q^{\max }, \quad \chi>\frac{\beta_{i k}^{i}}{\beta_{i, \text { min }}^{i}}, \\
q^{\max } \chi \frac{\beta_{i, \min }^{i}}{\beta_{i k}^{i}}, \quad \chi \leq \frac{\beta_{i k}^{i}}{\beta_{i, \min }^{i}},
\end{array}\right.
$$

Here, $\beta_{i, \text { min }}^{i}$ is defined as the smallest LSF value afforded by a user in the $i_{t h}$ cell serving by the BS in the $i_{t h}$ cell, which can be written as follows

$$
\beta_{i, \min }^{i}=\min \left(\beta_{i, 1}^{i}, \ldots, \beta_{i, k}^{i}, \ldots, \beta_{i, K}^{i}\right)
$$

This strategy ( provided in the equation 12 ) ) enables a user with the lowest channel condition to broadcast with maximum power. Besides, this strategy requires the other users to transmit with power limited to obey the constraint given through the Signal-to-Noise Ratio (SNR) ( i.e., the SNR can not exceed $10 d B$ higher at most). As the threshold $\chi$ is chosen equal to $10 d B$. Additionally, the maximum power of transmission $q^{\max }$ is equal to $10 \mathrm{dBm}$.

\section{Channel Estimation process}

In order to estimate the channel $\mathbf{H}_{i i k}$ at the $i_{t h}$ BS (i.e., $i_{t h}$ cell). A straightforward technique is adopted as referred to in [16,28, which represented as follows

$$
\hat{\mathbf{H}}_{i i k}^{L S}=\lambda_{i k}=\frac{\mathbf{y}_{i k}}{\sqrt{q_{i k}} N}=\mathbf{H}_{i i k}+\sum_{j \neq i}^{L} \sqrt{\frac{q_{j k}}{q_{i k}}} \mathbf{H}_{i j k}+\frac{w_{i k}}{\sqrt{q_{i k}} N}
$$

Here, $\lambda_{i k} \in \mathbb{C}^{M \times P}$ ( also referred to in some literature works as the CE based on LS [13], $\hat{\mathbf{H}}_{i i k}^{L S}$ ) is a matrix denoting the estimated version of channel $\mathbf{H}_{i i k}$ 
at the $i_{t h}$ BS using a traditional process. Furthermore, every column of $\lambda_{i k}$ is normally distributed $\left(\mathcal{C N}\left(0_{M}, \Lambda_{i k} \mathbf{I}_{\mathbf{M}}\right)\right)$, reads

$$
\Lambda_{i k}=\sum_{j=1}^{L} \frac{q_{j k}}{q_{i k}} \beta_{i j k}+\frac{1}{q_{i k} N}
$$

It is noteworthy that $\Lambda_{i k}$ integrates the LSF coefficients of all users that employ the same PS across all cells plus a parameter that represents the noise-power inverse.

\subsection{Ideal MMSE Channel Estimation}

The Bayesian-MMSE estimator is supposed that LSF coefficients are known a priori, which is not the case-in-fact in the practical implementation [16, 28. As the $i_{t h}$ BS can not have prior knowledge of the $k_{t h}$ user's LSF coefficients in the $j_{t h}$ cell $(i \neq j,\{i, j\}=1, \ldots, L)$ 6, 11. The estimated channel gain employing the MMSE estimator is expressed as follows 28

$$
\hat{\mathbf{H}}_{i i k}^{m m s e}=\frac{\beta_{i i k}}{\Lambda_{i k}} \lambda_{i k}=\frac{\beta_{i i k}}{\Lambda_{i k}} \hat{\mathbf{H}}_{i i k}^{L S}
$$

Here, each column of estimated channel gain $\hat{\mathbf{H}}_{i i k}^{m m s e}$ follows the normal distribution (i.e., $\left.\mathcal{N C}\left(0_{M}, \frac{\beta_{i i k}^{2}}{\Lambda_{i k}} \mathbf{I}_{M}\right)\right)$.

The MSE is the metric used for evaluating the performance of each estimator. Therefore, the MSE expression for Ideal MMSE is written as follows.

$$
\begin{aligned}
M S E_{i k}^{m m s e} & =\frac{1}{M P} \operatorname{Tr}\left\{\mathbb{E}\left\{\left\|\tilde{\mathbf{H}}_{i i k}^{m m s e}\right\|^{2}\right\}\right\}, \\
& =\frac{1}{M P} \operatorname{Tr}\left\{\mathbb{E}\left\{\left\|\mathbf{H}_{i i k}-\hat{\mathbf{H}}_{i i k}^{m m s e}\right\|^{2}\right\}\right\}, \\
& =\beta_{i i k}\left(1-\frac{\beta_{i i k}}{\Lambda_{i k}}\right)
\end{aligned}
$$

Here, $\tilde{\mathbf{H}}_{i i k}^{m m s e}$ symbolize the estimation error. The effectiveness of the ideal MMSE is strongly affected by the UL power $q_{i k}$, the desired LSF $\beta_{i i k}$, and the degree of inter-cell interference.

\subsection{Proposed Channel Estimation}

The Bayesian-MMSE estimator relies on a property that is not impossible to apply in a realistic context. Hence, This suggested estimator is investigated to overcome this issue by relying on the Maximum Likelihood (ML) estimator and using an unbiased estimator (i.e., $\mathbb{E}\{\hat{X}\}=X$ ) as mentioned in 28, 
where the suggested estimator is employed to estimate $\Lambda_{i k}$ rather than $\beta_{i i k}$. Thus, the complexity is reduced relative to the MMSE estimator since the MMSE estimator estimates individual LSF coefficients each. The estimate of the coefficient defined in equation $\sqrt{15}$ is denoted by $\hat{\Lambda}_{i k}$, and $\hat{\Lambda}_{i k}$ is estimated by relying on ML and the concept of unbiasedness (i.e., $\mathbb{E}\left\{\hat{\Lambda}_{i k}\right\}=\Lambda_{i k}$ ). The estimated coefficient $\hat{\Lambda}_{i k}$ is expressed as a function of $\lambda_{i k}$, NoA at the BS, $M$ and number of paths $P$ in the following form

$$
\hat{\Lambda}_{i k}=\frac{\left\|\lambda_{i k}\right\|_{F}^{2}}{M P}
$$

Here, $\|\bullet\|_{F}^{2}$ indicates the Frobenius norm, where $\left\|\lambda_{i k}\right\|_{F}^{2}=\operatorname{Tr}\left(\lambda_{i k}^{H} \lambda_{i k}\right)$ in which $\operatorname{Tr}(\bullet)$ indicates the Trace operator. $\mathbb{E}\left\{\lambda_{i k}^{H} \lambda_{i k}\right\}=M \Lambda_{i k} \mathbf{I}_{P}$ and according to equation 18), $M \hat{\Lambda}_{i k}=\frac{\left\|\lambda_{i k}\right\|_{F}^{2}}{P}$, i.e., as $\mathrm{P}$ increase the expression $\frac{\left\|\lambda_{i k}\right\|_{F}^{2}}{P}$ tends faster to the actual value $M \Lambda_{i k}$. By replacing $\Lambda_{i k}$ with the estimated expression (i.e., $\hat{\Lambda}_{i k}$ ) in the MMSE estimator expression, yielding the suggested estimator expression, which written as follows

$$
\mathbf{H}_{i i k}^{\text {suggested }}=M P \frac{\beta_{i i k}}{\left\|\lambda_{i k}\right\|_{F}^{2}} \lambda_{i k}
$$

According to the equation (19), the suggested estimator depends on the NoA at the BS and the number of paths per channel, where it is not the case for the ideal MMSE estimator. As we will illustrate by the mean of simulations in the coming section, an asymptotic result is obtained when $M$ or $P$ increases. It is important to note that in the single-path per channel situation (i.e., $\mathrm{P}$ $=1$ ), the equation $(19$ shortens to the equation (8) indicated in 13 . An approximate expression of the MSE for the suggested estimator is presented in the equation below

$$
\begin{aligned}
M S E_{i k}^{\text {suggested }} & =\frac{1}{M P} \operatorname{Tr}\left\{\mathbb{E}\left\{\left\|\tilde{\mathbf{H}}_{i i k}^{\text {suggested }}\right\|^{2}\right\}\right\} \\
& =\frac{1}{M P} \operatorname{Tr}\left\{\mathbb{E}\left\{\left\|\mathbf{H}_{i i k}-\hat{\mathbf{H}}_{\text {iik }}^{\text {suggested }}\right\|^{2}\right\}\right\} \\
& \approx \beta_{i i k}\left[1-\frac{\beta_{i i k}(M P-2)}{\Lambda_{i k}(M P-1)}\right]
\end{aligned}
$$

The effectiveness (i.e., the system performance is assessed using the MSE metric) of suggested estimator is strongly affected by the UL power $q$, the desired LSF $\beta_{i i k}$, NoA at the BS, $M$, number of paths for each channel, $P$ and the degree of inter-cell interference.

The table below is provided to recapitulate the parameter values utilized during the simulation result in this study. 


\begin{tabular}{|c|c|c|}
\hline Parameter & Description & parameter value \\
\hline $\mathrm{L}$ & Number of cell in the network & 7 \\
\hline $\mathrm{K}$ & Number of users in each cell & 10 \\
\hline $\mathrm{M}$ & $\begin{array}{c}\text { Number of BS antennas } \\
\text { (Excluding the Figure in which } \mathrm{M} \text { is varying) }\end{array}$ & 30 \\
\hline $\mathrm{N}$ & Length of PSs & 223 \\
\hline $\mathrm{P}$ & Number of paths for each channel & 20 \\
\hline- & Cell hole & $50 \mathrm{~m}$ \\
\hline- & Cell radius ( Micro Urban as mentioned in [22]) & $500 \mathrm{~m}$ \\
\hline $\mathrm{f}$ & Sequence Index & 119 \\
\hline$\alpha^{\operatorname{LoS}}$ & path-loss exponent for LoS & 2.6 \\
\hline$\alpha^{\operatorname{LoS}}$ & path-loss exponent for NLoS & 3.8 \\
\hline$\sigma^{\operatorname{LoS}}$ & shadowing standard deviation for LoS & 4 \\
\hline$\sigma^{N L O S}$ & shadowing standard deviation for NLoS & 10 \\
\hline
\end{tabular}

Table 1 The value of parameters used for simulation

\section{Simulation Results}

We adopt a multi-cell multi-user scenario assuming hexagonal-shape cells, the users are uniformly distributed over the cells, and the BSs are located in the cell center. The parameters' values used for the simulation are listed above in Table 1. In this segment, our objective is to affirm the theoretical expressions provided in the previous sections by exploiting simulation figures wherein the simulated and analytic results are plotted, analyzed, and compared in each setting. We deal with two estimators in this work, namely the Bayesian MMSE estimator and the suggested estimator. The suggested estimator is studied and analyzed in various circumstances to assert its effectiveness. We consider studying CE depending on how the communication between a user and its serving BS is. In other words, the communication user-BS can be obtained using either NLoS path only or using LoS and NLoS path depending on the environment, the distance between a user and its serving BS..., etc. The existence or absence of a LoS path has a significant change on the path-loss and shadowing values, which are assumed to be included in the scope of LSF. Thereby, the LSF model changes fundamentally. It is worth highlighting that the LSF coefficients are taken on average through 10000 realizations.

If the LoS path exists, the LSF coefficient given in equation (4) is performed, which characterizes with path-loss exponent $\alpha^{L o S}$ and standard deviation of shadowing effect $\sigma_{s f}^{L o S}$. While, if there is no LoS communication (i.e., only NLoS paths between the user and its serving BS occur), the LSF coefficient presented in equation (5) is performed, which characterizes with path-loss exponent $\alpha^{N L o S}$ and standard deviation of shadowing effect $\sigma_{s f}^{N L O S}$.

After having provided the value of parameters used for simulation results and determining the LSF model suitable for each situation. According to previous sections, the LoS path existence depends on the distance between the user and its serving BS. In other words, if the user-BS distance is inferior to $300 \mathrm{~m}$, hence, the user can benefit from the LoS path, as stated in [22]. Otherwise, 
the user can not benefit from the LoS path.

During this segment and in each situation, results analytic and simulated for each estimator are presented and compared.

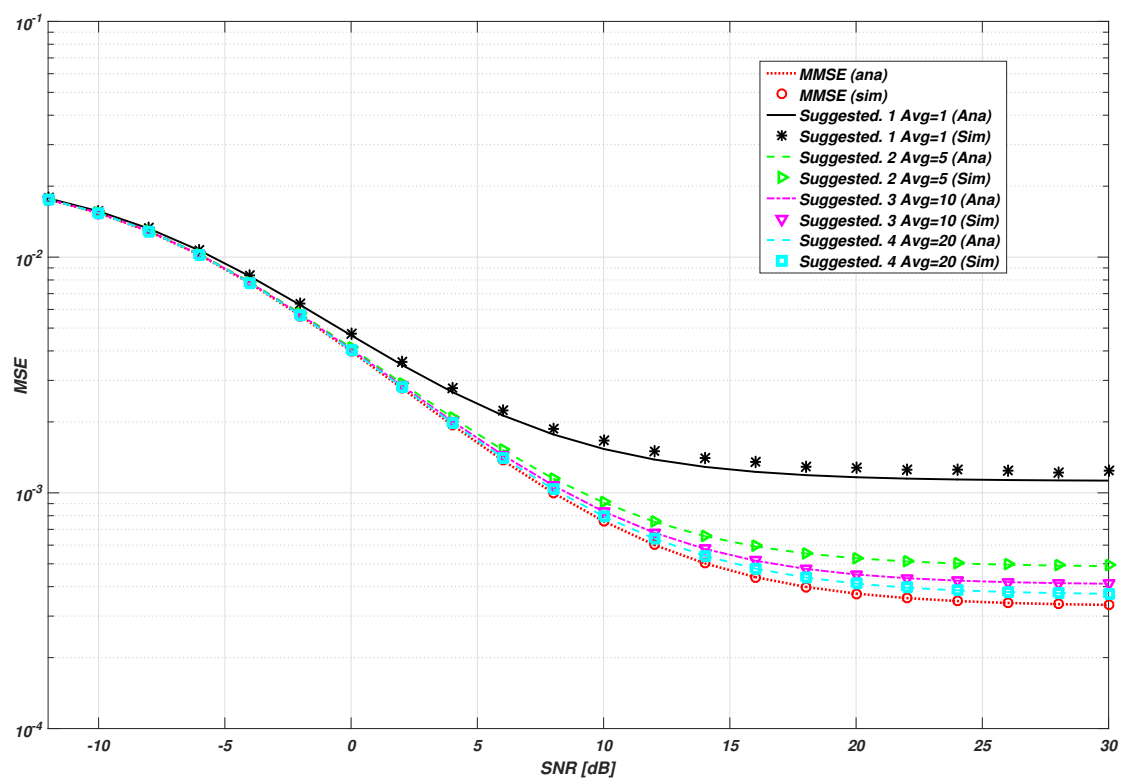

Fig. 1 MSE in function of $S N R(d B)$

Figure (1) illustrates the MSE metric with respect to SNR, as indicated above, the parameters used are presented in Table (1). The MMSE estimator's performance is compared to the suggested estimator's performance. As forecast, the estimation quality presented through the MMSE estimator is the best compared to the suggested estimator. on the flip side, considering different averaged components of the square matrix $\lambda_{i k}^{H} \lambda_{i k}$ from the main diagonal in order to evaluate the estimation quality presented by the suggested estimator. From figure (1), one can notice that the effectiveness of the suggested estimator is strongly related to the Number of Averaged Elements (NoAE) that are selected. In other words, when a small NoAE is identified, a large MSE gap between the MMSE estimator and the suggested estimator is created. This gap is considerably smaller as the NoAE increase. In addition, we provide analytical results and simulated results for each estimator to compare its analytical and simulated performances. According to the figure, we conclude that the analytic results and the simulated results perform similarly.

Figure (2) is displayed to evaluate how the NoAE can influence the efficiency of the suggested estimator, where the suggested estimator's performance is strongly related to the NoAE that are selected. According to the Figure (2), which illustrate the MSE metric in function of the NoA at the BS, M. The 


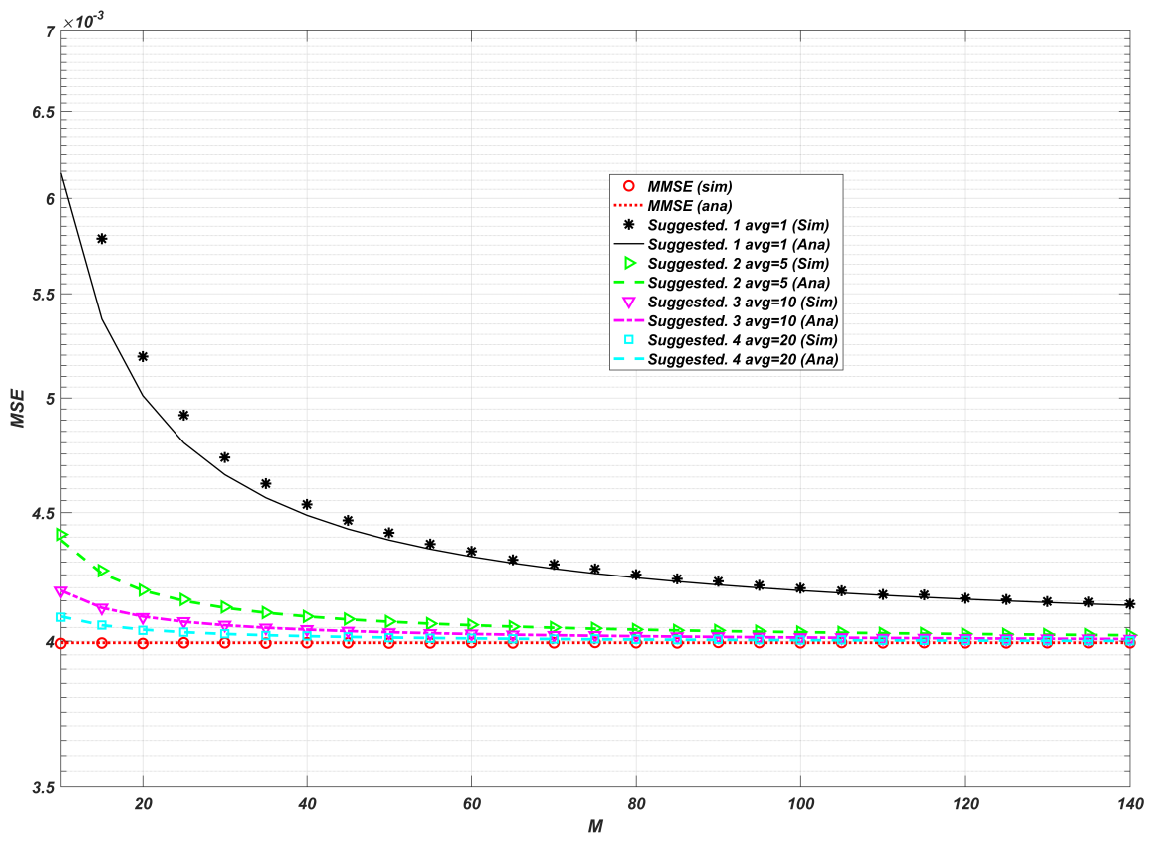

Fig. 2 MSE in function of the number of BS antennas

estimation quality presented through the MMSE estimator is compared to the suggested estimator. As expected, compared to the suggested estimator, the performance of the MMSE estimator is better. In order to determine the performance of the suggested estimator, various average components of the square matrix $\lambda_{i k}^{H} \lambda i k$ are taking from the main diagonal. One can remark that the NoAE chosen has a high effect on the efficiency of the estimator suggested. Otherwise stated, a small NoAE provide a large MSE gap between the MMSE estimator and the suggested estimator. This gap is relatively large when a small NoA is operating at the BS. By increasing the NoAE and the NoA at the BS, this gap tends to be very small. Additionally, better performance (i.e., MSE values of the suggested estimator very near the MSE values of the MMSE estimator ) is achieving when the NoAE is equal to the number of multi-path components. Furthermore, figure (2) displays analytical results and simulated results for each estimator. The analysis provided above is valid for both situations since they perform similarly. Notice that the case in which we employ a small NoAE provides a small gap between analytical results and simulated results. Indeed, the reason for this is that only a small NoAE are employed.

Figure (3) introduced the MSE metric against the number of multi-path, $P$, against the PS length, $N$, for the MMSE estimator and suggested estimator, where the $S N R$ is setting to $20 d B$. This Figure is affirmed the theoretical inequality, namely $N \geqslant K P$. Otherwise stated, increasing paths number leads 


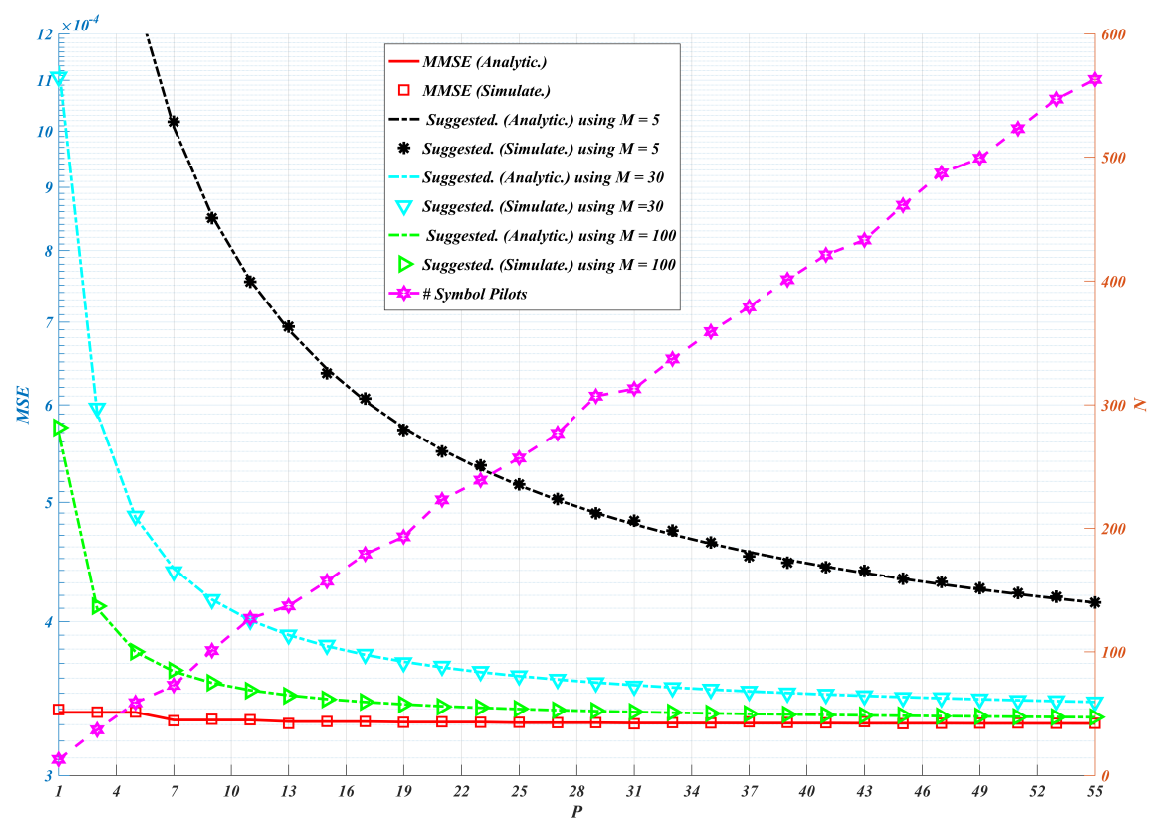

Fig. 3 MSE in function of both pilot length and paths number

to an increase in the PS length to serving $\mathrm{K}$ users with an orthogonal PS. Furthermore, the BS can not estimate the channel without relying on orthogonality among PSs. From figure (3), the effectiveness of the suggested estimator is strongly related to the NoA at the BS and the number of multipath chosen (recall that the paths number is obeying the limited coherence block constraints). As the number of BS antennas increase, the performance of the suggested estimator is greatly enhanced. One can notice that the MMSE estimator keeps small MSE values compared to the suggested estimator for all values of multi-path, $\mathrm{P}$. on the flip side, we provide analytical and simulated results for each estimator. Hence, we concluded that the analytical and simulated results perform similarly for each estimator.

\section{Conclusion}

A straightforward channel estimator has suggested through this work for multipath communication between each pair of antennas at both ends. The suggested estimator has performed to overcome the unrealistic and inapplicable property on which the MMSE estimator is relied, which can be explained by the fact that the MMSE estimator has taken into account foreknowledge of the LSF coefficients of the interfering users. Besides, we overcame this problem in a more real propagation environment in which multi-path channels are realized either using NLoS only or using LOS and NLoS paths depending on 
the distance at which the user is located from his serving BS. Additionally, we have introduced a PoC policy so that the users at the cell edge are less affected by the PC problem since we have considered the worst-case scenario in which frequency reuse is 1 ( the desired cell suffers from high interference from all other cells in the network ). In summary, the suggested estimator has succeeded overcoming the unrealistic property provided by the MMSE estimator since it displays asymptotic behavior to the performance of the MMSE estimator in all studied situations. Besides, the analytical and simulated results have performed similarly in all the analyzed plots.

\section{References}

1. Andreas F Molisch, Vishnu V Ratnam, Shengqian Han, Zheda Li, Sinh Le Hong Nguyen, Linsheng Li, and Katsuyuki Haneda. Hybrid beamforming for massive mimo: A survey. IEEE Communications magazine, 55(9):134-141, 2017.

2. Tadilo Endeshaw Bogale and Long Bao Le. Beamforming for multiuser massive mimo systems: Digital versus hybrid analog-digital. In 2014 IEEE Global Communications Conference, pages 4066-4071. IEEE, 2014.

3. Thomas L Marzetta. Noncooperative cellular wireless with unlimited numbers of base station antennas. IEEE transactions on wireless communications, 9(11):3590-3600, 2010.

4. Fredrik Rusek, Daniel Persson, Buon Kiong Lau, Erik G Larsson, Thomas L Marzetta, Ove Edfors, and Fredrik Tufvesson. Scaling up mimo: Opportunities and challenges with very large arrays. IEEE signal processing magazine, 30(1):40-60, 2012.

5. Erik G Larsson, Ove Edfors, Fredrik Tufvesson, and Thomas L Marzetta. Massive mimo for next generation wireless systems. IEEE communications magazine, 52(2):186-195, 2014.

6. Jubin Jose, Alexei Ashikhmin, Thomas L Marzetta, and Sriram Vishwanath. Pilot contamination and precoding in multi-cell tdd systems. IEEE Transactions on Wireless Communications, 10(8):2640-2651, 2011.

7. Hien Quoc Ngo, Thomas L Marzetta, and Erik G Larsson. Analysis of the pilot contamination effect in very large multicell multiuser mimo systems for physical channel models. In 2011 IEEE international conference on acoustics, speech and signal processing (ICASSP), pages 3464-3467. IEEE, 2011.

8. Trinh Van Chien and Emil Björnson. Massive mimo communications. In $5 G$ Mobile communications, pages 77-116. Springer, 2017.

9. Emil Björnson, Jakob Hoydis, and Luca Sanguinetti. Massive mimo networks: Spectral, energy, and hardware efficiency. Foundations and Trends in Signal Processing, 11(34):154-655, 2017.

10. Jakob Hoydis, Stephan Ten Brink, and Mérouane Debbah. Massive mimo in the ul/dl of cellular networks: How many antennas do we need? IEEE Journal on selected Areas in Communications, 31(2):160-171, 2013.

11. Hien Quoc Ngo, Erik G Larsson, and Thomas L Marzetta. The multicell multiuser mimo uplink with very large antenna arrays and a finite-dimensional channel. IEEE Transactions on Communications, 61(6):2350-2361, 2013.

12. Haifan Yin, David Gesbert, Miltiades Filippou, and Yingzhuang Liu. A coordinated approach to channel estimation in large-scale multiple-antenna systems. IEEE Journal on selected areas in communications, 31(2):264-273, 2013.

13. Amin Khansefid and Hlaing Minn. On channel estimation for massive mimo with pilot contamination. IEEE Communications Letters, 19(9):1660-1663, 2015.

14. Thomas L Marzetta and Hien Quoc Ngo. Fundamentals of massive MIMO. Cambridge University Press, 2016. 
15. Felipe AP de Figueiredo, Fabbryccio ACM Cardoso, Ingrid Moerman, and Gustavo Fraidenraich. Channel estimation for massive mimo tdd systems assuming pilot contamination and flat fading. EURASIP Journal on Wireless Communications and Networking, 2018(1):1-10, 2018.

16. Theodore S Rappaport et al. Wireless communications: principles and practice, volume 2. prentice hall PTR New Jersey, 1996.

17. Nafiseh Shariati, Emil Björnson, Mats Bengtsson, and Mérouane Debbah. Lowcomplexity polynomial channel estimation in large-scale mimo with arbitrary statistics. IEEE Journal of Selected Topics in Signal Processing, 8(5):815-830, 2014.

18. FA Pereira de Figueiredo, DA Mendes Lemes, C Ferreira Dias, and Gustavo Fraidenraich. Massive mimo channel estimation considering pilot contamination and spatially correlated channels. Electronics Letters, 56(8):410-413, 2020.

19. Özgecan Özdogan, Emil Björnson, and Erik G Larsson. Massive mimo with spatially correlated rician fading channels. IEEE Transactions on Communications, 67(5):3234$3250,2019$.

20. Thomas L Marzetta. How much training is required for multiuser mimo? In 2006 Fortieth Asilomar Conference on Signals, Systems and Computers, pages 359-363. IEEE, 2006 .

21. Giuseppe Caire, Nihar Jindal, Mari Kobayashi, and Niranjay Ravindran. Multiuser mimo achievable rates with downlink training and channel state feedback. IEEE Transactions on Information Theory, 56(6):2845-2866, 2010.

22. 3rd generation partnership project, technical specification group radio access network; spatial channel model for multiple input multiple output (mimo) simulations. 3GPP TR 25.996 V14.0.0, 2017.

23. Bernard Sklar. Rayleigh fading channels in mobile digital communication systems. i. characterization. IEEE Communications magazine, 35(7):90-100, 1997.

24. Erik Dahlman, Stefan Parkvall, and Johan Skold. 4G, LTE-advanced Pro and the Road to 5G. Academic Press, 2016.

25. David Chu. Polyphase codes with good periodic correlation properties (corresp.). IEEE Transactions on information theory, 18(4):531-532, 1972.

26. Jae Won Kang, Younghoon Whang, Byung Hoon Ko, and Kwang Soon Kim. Generalized cross-correlation properties of chu sequences. IEEE transactions on information theory, 58(1):438-444, 2011.

27. 3rd generation partnership project technical specification group radio access network evolved universal terrestrial radio access (e-utra) physical channels and modulation (release 11). TS 36.211 ver.11.0.0, 2012.

28. Steven M Kay. Fundamentals of statistical signal processing. Prentice Hall PTR, 1993. 